\title{
High-Energy Spectral Signatures in Gamma-Ray Bursts
}

\author{
Matthew G. Baring ${ }^{\dagger}$ \\ Laboratory for High Energy Astrophysics, Code 661 \\ NASA Goddard Space Flight Center, Greenbelt, MD 20771 \\ baring@lheavx.gsfc.nasa.gov \\ ${ }^{\dagger}$ Universities Space Research Association
}

\begin{abstract}
One of the principal results obtained by the EGRET experiment aboard the Compton Gamma-Ray Observatory (CGRO) was the detection of several $\gamma$-ray bursts (GRBs) above $100 \mathrm{MeV}$. The broad-band spectra obtained for these bursts gave no indication of any high energy spectral attenuation that might preclude detection of bursts by ground-based Čerenkov telescopes (ACTs), thus motivating several TeV observational programs. This paper explores the expectations for the spectral properties in the $\mathrm{TeV}$ and sub- $\mathrm{TeV}$ bands for bursts, in particular how attenuation of photons by pair creation internal to the source modifies the spectrum to produce distinctive spectral signatures. The energy of spectral breaks and the associated spectral indices provide valuable information that can constrain the bulk Lorentz factor of the GRB outflow at a given time. These characteristics define palpable observational goals for $\mathrm{ACT}$ programs, and strongly impact the observability of bursts in the $\mathrm{TeV}$ band.
\end{abstract}

\section{INTRODUCTION}

High energy gamma-rays have been observed for six gamma-ray bursts by the EGRET experiment on CGRO. Most conspicuous among these observations is the emission of an $18 \mathrm{GeV}$ photon by the GRB940217 burst [1]. Taking into account the instrumental field of view, these detections indicate that emission in the $1 \mathrm{MeV}-10$ $\mathrm{GeV}$ range is probably common among bursts, if not universal. One implication of GRB observability at energies around or above $1 \mathrm{MeV}$ is that, at these energies, spectral attenuation by two-photon pair production $\left(\gamma \gamma \rightarrow e^{+} e^{-}\right)$is absent in the source. From this fact, early on Schmidt [2] concluded that if a typical burst produced quasi-isotropic radiation, it had to be less distant than a few kpc, since the optical depth $\tau_{\gamma \gamma}$ scales as the square of the distance to the burst.

This result conflicted with BATSE's determination of the spatial isotropy and inhomogeneity of bursts [3], which suggested that they are either in an extended halo or at cosmological distances (where $\tau_{\gamma \gamma} \sim 10^{12}$ for isotropic photons). Hence Fenimore et al. [4] proposed that GRB photon angular distributions are highly beamed and produced by a relativistically moving plasma, a suggestion that has 
become very popular. This can dramatically reduce $\tau_{\gamma \gamma}$ and blueshift spectral attenuation turnovers out of the observed spectral range. Various determinations of the bulk Lorentz factor $\Gamma$ of the GRB medium have been made in recent years, mostly concentrating $[5,6]$ on cases where the angular extent of the source was of the order of $1 / \Gamma$. These calculations generally assume an infinite power-law burst spectrum, and deduce [7] that gamma-ray transparency up to the maximum energy detected by EGRET requires $\Gamma \gtrsim 100-10^{3}$ for cosmological bursts.

While power-law source spectrum assumption is expedient, the spectral curvature seen in most GRBs by BATSE [8] is expected to play an important role in reducing the opacity for potential TeV emission from these sources (Baring \& Harding [9]). Such curvature is patently evident in $200 \mathrm{keV}-2 \mathrm{MeV}$ spectra of some EGRETdetected bursts, and its prevalence in bursts is indicated by the generally steep EGRET spectra for bursts $[1,10,11]$. In this paper, the principal effects introduced into pair production opacity calculations by spectral breaks in the BATSE energy range are considered, focusing the work of [9] to identify the properties of cosmological bursts in the $1 \mathrm{GeV}-1 \mathrm{TeV}$ range. These signatures are clearly distinguishable from absorption by background radiation fields, thereby defining diagnostics that future ground-based initiatives such as Veritas, MILAGRO, HESS and MAGIC, and space missions such as GLAST can provide for GRB studies.

\section{SPECTRAL CURVATURE AND $\gamma-\gamma$ ATTENUATION}

The simplest picture $[5,6]$ of relativistic beaming has "blobs" of material moving with a bulk Lorentz factor $\Gamma$ more-or-less toward the observer, and having an angular "extent" $\sim 1 / \Gamma$. For an infinite power-law spectrum $n(\varepsilon)=n_{\gamma} \varepsilon^{-\alpha}$, where $\varepsilon$ is the photon energy in units of $m_{e} c^{2}$ (a dimensionless convention used throughout), for which the optical depth to pair creation assumes the form $\tau_{\gamma \gamma}(\varepsilon) \propto$ $\varepsilon^{\alpha-1} \Gamma^{-(1+2 \alpha)}$ for $\Gamma \gg 1$. As noted above, the input source spectrum needs to be modified, to explore the effects of a relative depletion of low energy photons in the BATSE range. The simplest approximation to spectral curvature is a power-law broken at a dimensionless energy $\varepsilon_{\mathrm{B}}\left(=E_{\mathrm{B}} / 0.511 \mathrm{MeV}\right)$ :

$$
n(\varepsilon)=n_{\gamma} \varepsilon_{\mathrm{B}}^{-\alpha_{h}}\left\{\begin{array}{ll}
\varepsilon_{\mathrm{B}}^{\alpha_{l}} \varepsilon^{-\alpha_{l}}, & \text { if } \varepsilon \leq \varepsilon_{\mathrm{B}} \\
\varepsilon_{\mathrm{B}}^{\alpha_{h}} \varepsilon^{-\alpha_{h}}, & \varepsilon>\varepsilon_{\mathrm{B}} .
\end{array},\right.
$$

The optical depth determination for such a distribution utilizes results obtained in [12] for truncated power-laws. The resulting forms are presented in [9], and the optical depth $\tau_{\gamma \gamma}(\varepsilon)$ for attenuation of a broken power-law photon distribution has the basic form

$$
\frac{\tau_{\gamma \gamma}(\varepsilon)}{n_{\gamma} \sigma_{\mathrm{T}} R} \propto\left\{\begin{array}{ll}
\frac{\varepsilon^{\alpha_{h}-1}}{\Gamma^{2 \alpha_{h}}}, & \text { if } \varepsilon \lesssim \Gamma^{2} / \varepsilon_{\mathrm{B}}
\end{array},\right.
$$




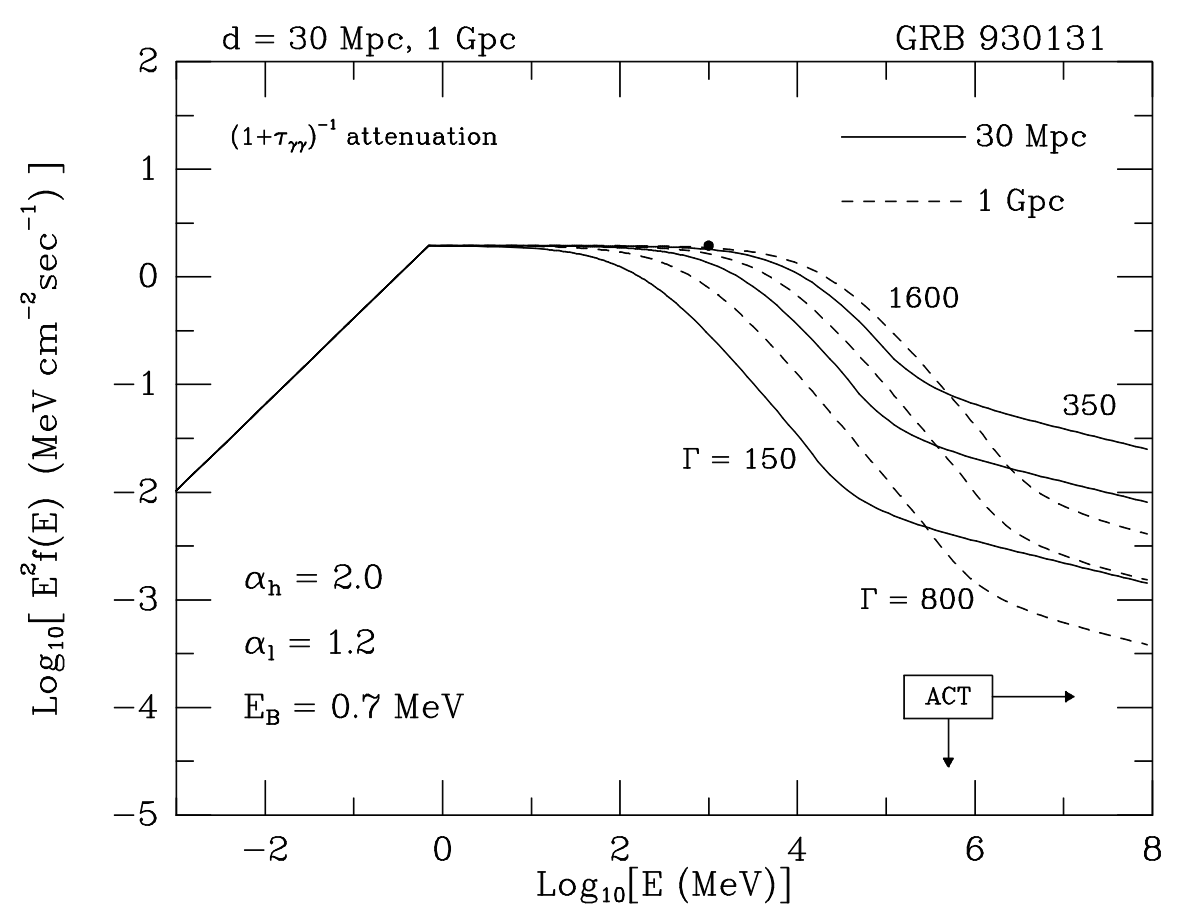

FIGURE 1. The $\gamma-\gamma$ attenuation, internal to the source, for GRB 930131 at distances typical of nearby (solid curves, $\Gamma=150,250,350$ ) and distant (short dashed curves, $\Gamma=800,1200,1600$ ) cosmological origin, for bulk Lorentz factors $\Gamma$ of the emitting region. The source spectrum $\left(\nu F_{\nu}\right.$ format) was a power-law broken at $E_{\mathrm{B}}=0.7 \mathrm{MeV}$, with spectral indices $\alpha_{l}=1.2$ and $\alpha_{h}=2.0$. The filled circle denotes the highest energy EGRET photon at $1000 \mathrm{MeV}$ [7]. The threshold and sensitivity for ACT observations of later bursts is indicated by the "ACT" box.

that implies breaks in the absorbed portion of the hard gamma-ray spectrum that "image" the BATSE band break in the seed photons. More gradual spectral curvature can be treated by fitting the GRB continuum with piecewise continuous power-laws. A variability "size" $R_{v}=3 \times 10^{7} \mathrm{~cm}(=R / \Gamma)$ is chosen here following $[7,9]$, and the observed flux at $1 \mathrm{MeV}$ normalizes the source density coefficient $n_{\gamma}$.

The results of the attenuation of the spectra in Eq. (1) are depicted in Fig. 1 using an attenuation factor $1 /\left(1+\tau_{\gamma \gamma}\right)$ that is appropriate for opacity skin depth effects. The source spectrum parameters are chosen to approximate the observed values for the "Superbowl burst" GRB930131, for two different extragalactic distance scales: the nearer one, $30 \mathrm{Mpc}$, is appropriate to scenarios where GRBs generate ultra-high energy cosmic rays. Clearly the attenuation is marked in the $\mathrm{GeV}-\mathrm{TeV}$ band for the Lorentz factors $\Gamma$ chosen, and could be reduced by increasing $\Gamma$. The onset of attenuation couples to $\Gamma$ and the EGRET band spectral index $\alpha_{h}$, and above this turnover the immediate spectral index is $1-2 \alpha_{h}$. Precise knowledge of the GRB distance, such as through redshifts of accompanying optical afterglows, would facilitate the determination of tight constraints on $\Gamma$. For either distance scale in Fig. 1, there is a flattening (to index $1-\alpha_{h}-\alpha_{l}$ ) in the $\mathrm{TeV} / \mathrm{sub}-\mathrm{TeV}$ band that is a consequence of the spectral break in the BATSE band: it arises at $\varepsilon \sim \Gamma^{2} / \varepsilon_{\mathrm{B}}$. 


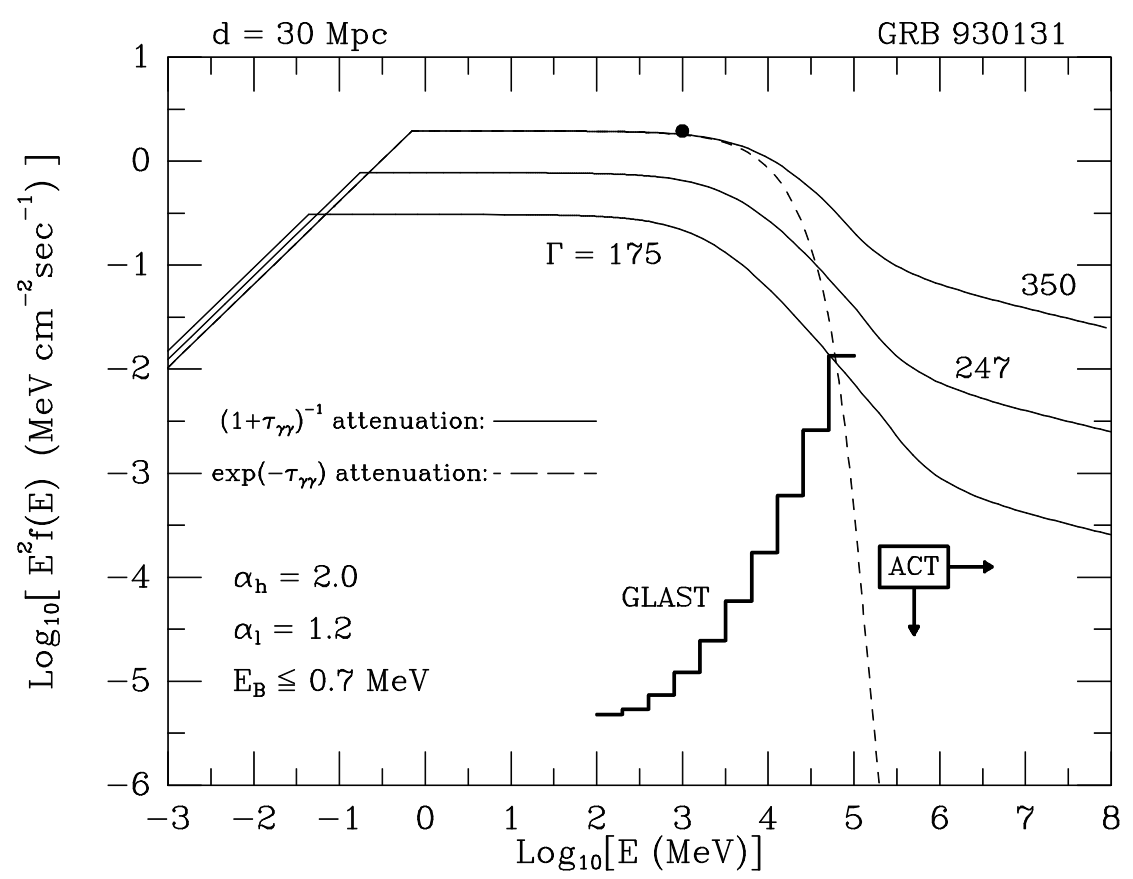

FIGURE 2. An evolutionary sequence for $\gamma-\gamma$ attenuation, starting with a GRB930131 data fit, appropriate to an adiabatically-decelerating blast wave. The GLAST steady-source differential ( $\Delta E / E=2$ step-function) and approximate Whipple integral sensitivities (ACT box; derived from upper limits to later bursts) are depicted. A case of exponential attenuation (for the $\Gamma=350$, dashed line) is illustrated; it would inhibit detections by ACTs.

The potential for observational diagnostics is immediately apparent. First, the extant EGRET data already provides a lower bound to $\Gamma$ : the dot on Fig. 1 represents the highest energy photon from GRB930131, and clearly suggests that $\Gamma \gtrsim 250$ for $d=30 \mathrm{Mpc}$ or $\Gamma \gtrsim 800$ for $d=1 \mathrm{Gpc}$. Second, the sensitivity of ACTs is easily sufficient to detect bursts even with significant attenuation, so that they could well probe the spectral issues raised here. While the Whipple rapid search [13] postdated the EGRET detections, and produced merely upper limits as indicated in Fig. 1, an intriguing possible detection of a BATSE burst by the MILAGRITO forerunner to MILAGRO was announced at this meeting by McEnery et al. (these proceedings), foreshadowing advances to come.

Perhaps the greatest strides in understanding will be precipitated by broad-band spectral coverage afforded by simultaneous detection of bursts by GLAST and TeV experiments like MILAGRO. Fig. 2 displays a time-evolutionary sequence of GRB spectra, including the effects of $\gamma-\gamma$ attenuation, and compares this with the potentially-constraining current Whipple integral sensitivity threshold (deduced from the results of [13]), and the projected GLAST steady-source differential sensitivity. The GLAST sensitivity is obtained from simulations (Digel, private communication) of the spectral capability for high latitude, steady sources in a one-year survey, i.e. roughly 8 weeks on source. The real GLAST sensitivity for transient GRBs of duration $t_{\text {dur }}$ can be estimated to be roughly $\left[(8 \text { weeks }) / t_{\text {dur }}\right]^{1 / 2}$ times that 
depicted. Note that the differential sensitivity is the most appropriate measure for spectral diagnostic capabilities. Evidently, ACTs and GLAST working in concert will be able to determine the spectral shape and evolution of bright, flat-spectrum bursts like GRB930131 if the attenuation is no more dramatic than $1 /\left(1+\tau_{\gamma \gamma}\right)$. The particular evolutionary scenario depicted in Fig. 2 is an adiabatic one for blast wave deceleration during the sweep-up phase, where the dependences on time $t$

are $\Gamma \propto t^{-3 / 8}, \varepsilon_{\mathrm{B}} \propto \Gamma^{4} \propto t^{-3 / 2}$, and $\varepsilon_{\mathrm{B}}^{2} f\left(\varepsilon_{\mathrm{B}}\right) \propto \Gamma^{8 / 3} \propto t^{-1}$ for the flux at the peak [14]. Shifts in the turnover energy and sub-TeV break energy, and correlations with BATSE flux and break energy should be discernible in bright sources.

It must be emphasized that these internal absorption characteristics are easily distinguishable from those of external absorption due to the cosmological infrared background along the line of sight $[15,16]$. Attenuation by such background fields couples to the redshift, not parameters internal to the source nor the shape of the spectrum in the BATSE and EGRET bands. Furthermore, it is always exponential in nature (i.e. of severity equivalent to the dashed curve in Fig. 2) since the emission region is distinct from the location of the soft target photons, and is patently independent of time. The possibility of confusing such with the internal attenuation that forms the focus of this paper seems minimal. Hence, the prospects for powerful spectral diagnostics in bright bursts with atmospheric Cerenkov telescopes and the GLAST mission promise an exciting time ahead for the field of high energy gamma-ray astronomy.

Acknowledgments: I thank Alice Harding and Brenda Dingus for helpful discussions, and Seth Digel for simulating GLAST spectral sensitivities.

\section{REFERENCES}

1. Hurley, K. et al. Nature 372, 652 (1994).

2. Schmidt, W. K. H. Nature 271, 525 (1978).

3. Meegan, C., et al. Ap. J. Supp. 106, 65 (1996).

4. Fenimore, E. E., Epstein, R. I. \& Ho, C. in Gamma-Ray Bursts, eds. Paciesas, W. S. and Fishman, G. J., (AIP, New York) p. 158 (1992).

5. Krolik, J. H. \& Pier, E. A. Ap. J. 373, 277 (1991).

6. Baring, M. G. Ap. J. 418, 391 (1993)

7. Baring, M. G. \& Harding, A. K. Ap. J. 491, 663 (1997b).

8. Band, D., et al. Ap. J. 413, 281 (1993).

9. Baring, M. G. \& Harding, A. K. Ap. J. Lett. 481, L85 (1997a).

10. Schneid, E. J., et al. Astron. Astr. (Lett.) 255, L13 (1992).

11. Sommer, M., et al. Ap. J. Lett. 422, L63 (1994).

12. Gould, R. J. \& Schreder, G. P. Phys. Rev. 155, 1404 (1967).

13. Connaughton, V. et al. Ap. J. 479, 859 (1997).

14. Dermer, C. D., Chiang, J. \& Böttcher, M. Ap. J. 513, 656 (1999).

15. Stecker, F. W. \& De Jager, O. C. Space Sci. Rev. 75, 401 (1996).

16. Mannheim, K., Hartmann, D. \& Funk, B. Ap. J. 467, 532 (1996). 